\title{
Characterisation of a PERCIVAL monolithic active pixel prototype using synchrotron radiation
}

J. Correa, ${ }^{a, b, 1}$ M. Bayer, ${ }^{a, b}$ P. Göttlicher, ${ }^{a}$ S. Lange, ${ }^{a, b}$ A. Marras, ${ }^{a, b}$ M. Niemann, ${ }^{a, b}$

S. Reza, ${ }^{a, c}$ I. Shevyakov, ${ }^{a}$ S. Smoljanin,,${ }^{a, b}$ M. Tennert, ${ }^{a, b}$ Q. Xia, ${ }^{a}$ M. Viti, ${ }^{a, b}$

C.B. Wunderer,,${ }^{a, b}$ M. Zimmer, ${ }^{a}$ D. Dipayan, ${ }^{f}$ N. Guerrini, ${ }^{f}$ B. Marsh, ${ }^{f}$ I. Sedgwick, ${ }^{f}$

R. Turchetta, ${ }^{f}$ G. Cautero, ${ }^{d}$ D. Giuressi, ${ }^{d}$ A. Khromova, ${ }^{d}$ G. Pinaroli, ${ }^{d}$ R. Menk, ${ }^{d}$ L. Stebel, ${ }^{d}$

R. Fan, ${ }^{e}$ J. Marchal, ${ }^{e}$ U. Pedersen, ${ }^{e}$ N. Rees, ${ }^{e}$ P. Steadman, ${ }^{e}$ M. Sussmuth, ${ }^{e}$ N. Tartoni, ${ }^{e}$

H. Yousef, ${ }^{e}$ H.J. Hyun, ${ }^{g}$ K. Kim, ${ }^{g}$ S. Rah ${ }^{g}$ and H. Graafsma ${ }^{a, b, c}$

${ }^{a}$ DESY (Deutsches Elektronensynchrotron), Notkestr. 85, 22607 Hamburg, Germany

${ }^{b}$ CFEL (Center for Free-Electron Laser Science), Luruper Ch. 149, 22607 Hamburg, Germany

${ }^{c}$ Mid Sweden University, Holmgatan 10, 85170 Sundsvall, Sweden

${ }^{d}$ Elettra Sincrotrone Trieste, S.S. 14 km 163.5, 34149 Basovizza, Trieste, Italy

${ }^{e}$ Diamond Light Source, Didcot OX 11 ODE, U.K.

${ }^{f}$ Rutherford Appleton Laboratory / STFC, Didcot OX 11 OQX, U.K.

${ }^{g}$ Pohang Accelerator Laboratory, Jigokro-127-beongil, 790834 Pohang, Korea

E-mail: Jonathan.Correa@desy.de

Abstract: PERCIVAL ("Pixelated Energy Resolving CMOS Imager, Versatile And Large") is a monolithic active pixel sensor (MAPS) based on CMOS technology. Is being developed by DESY, RAL/STFC, Elettra, DLS, and PAL to address the various requirements of detectors at synchrotron radiation sources and Free Electron Lasers (FELs) in the soft X-ray regime. These requirements include high frame rates and FELs base-rate compatibility, large dynamic range, single-photon counting capability with low probability of false positives, high quantum efficiency (QE), and (multi-)megapixel arrangements with good spatial resolution. Small-scale back-sideilluminated (BSI) prototype systems are undergoing detailed testing with X-rays and optical photons, in preparation of submission of a larger sensor.

A first BSI processed prototype was tested in 2014 and a preliminary result — first detection of $350 \mathrm{eV}$ photons with some pixel types of PERCIVAL — reported at this meeting a year ago.

Subsequent more detailed analysis revealed a very low QE and pointed to contamination as a possible cause. In the past year, BSI-processed chips on two more wafers were tested and their

${ }^{1}$ Corresponding author. 
response to soft X-ray evaluated. We report here the improved charge collection efficiency (CCE) of different PERCIVAL pixel types for $400 \mathrm{eV}$ soft X-rays together with Airy patterns, response to a flat field, and noise performance for such a newly BSI-processed prototype sensor.

KEYwORDS: X-ray detectors; Instrumentation for FEL; Solid state detectors 


\section{Contents}

1 Introduction 1

2 The PERCIVAL concept and sensors 2

2.1 The concept 2

2.2 Prototype sensors 5

3 Testing PERCIVAL prototypes 5

$\begin{array}{lll}3.1 \text { Noise } & 6\end{array}$

$\begin{array}{ll}3.2 & \text { Uniform illumination } \\ 3.3 & \text { Sensitivity }\end{array}$

$\begin{array}{lll}3.3 & \text { Sensitivity } & 6\end{array}$

$\begin{array}{ll}3.4 & \text { Charge Collection Efficiency }\end{array}$

4 Summary and outlook $\quad 8$

\section{Introduction}

The growing luminosity of the 3rd-generation synchrotron as well as the advent of the Free Electron Laser (FEL) sources have significantly increased the demands on X-ray detector systems.

The high photon intensities of a single FEL pulse result in diffraction patterns which can reach $10^{5}$ photons/pixel/frame and more. At the same time, other regions within the same pattern demand single-photon counting capabilities with high confidence in false positive rejection. As a consequence, both low noise and high dynamic range are required. Moreover, to cope with the repetition rate of these novel sources, new detector systems need to work at frame rates up to $120 \mathrm{~Hz} .{ }^{1}$ Since all photons arrive in time windows as short as tens of femtoseconds, photon counting is not feasible and the use of integrating detectors is imperative.

Also, large and uninterrupted imaging areas combined with small pixel sizes and a high Quantum Efficiency (QE) over a large photon energy range is commonly required.

A collaboration of different Photon Science institutions (DESY, Elettra, Diamond, and PAL) has joined forces with RAL/STFC to develop a new system to meet these challenges for the soft X-ray energy regime (250 eV - $1 \mathrm{keV}$ ): PERCIVAL ("Pixelated Energy Resolving CMOS Imager, Versatile and Large").

${ }^{1}$ Note that this is only the base rate (e.g. $10 \mathrm{~Hz}$ for FLASH and Fermi, $60 \mathrm{~Hz}$ for SACLA, and $120 \mathrm{~Hz}$ for LCLS). In order to cope with the bunch rate within the multi-bunch pulses at FLASH or EU-XFEL, MHz frame rates detectors would be required [1].) 
Table 1. Detector performance aim.

\begin{tabular}{|l|l|}
\hline Energy Range & $\begin{array}{l}\text { Primary: } 250-1,000[\mathrm{eV}] \\
\text { Extended: } 100-3000[\mathrm{eV}]\end{array}$ \\
\hline QE over Primary Energy Range & $>85 \%$, uniform over pixel \\
\hline Frame Rate & $(1-) 120[\mathrm{~Hz}]$ \\
\hline Pixel Size & $27($ prototypes: 25$)[\mu \mathrm{m}]$ \\
\hline Sensor Size & $2 \mathrm{M}-1408 \times 1484$ pixels, $4 \times 4\left[\mathrm{~cm}^{2}\right]$ \\
& $13 \mathrm{M}-3520 \times 3710$ pixels, $10 \times 10\left[\mathrm{~cm}^{2}\right]$ \\
\hline Noise RMS & $<15\left[\mathrm{e}^{-}\right]$ \\
\hline "Full Well" & $>10^{7}\left[\mathrm{e}^{-}\right]$ \\
\hline Resulting Dynamic Range & $10^{5}[\mathrm{ph}]($ $250 \mathrm{eV})$ \\
\hline Sensor Output & Digital, LVDS \\
\hline Buttability & 2 -side $($ adjacent edges $)$ \\
\hline Exposure Modes & FEL - all photons in $<300 \mathrm{fs}$ \\
& Synchrotron - quasi-continuous \\
\hline
\end{tabular}

\section{The PERCIVAL concept and sensors}

For the described needs, CMOS sensors offer several advantages compared to other available technologies: they can be faster than CCDs since their architecture naturally enables massive parallelisation; the use of smaller pixels is less problematic than in hybrid systems because it does not push the limits of bump bonding technology and finally, smaller photo-diode capacitances can be achieved in CMOS compared to hybrid pixel sensors allowing lower noise levels.

A closer look at the target detector performances is taken in this section. Some of the most important are summarised in table 1. This work will mainly focus on the sensor itself: pixel, gain switching mechanism, and ADCs. A detailed description of the full PERCIVAL system has been given elsewhere [2].

\subsection{The concept}

The PERCIVAL soft X-ray imager has a primary energy range between $250 \mathrm{eV}$ and $1 \mathrm{keV}$. Over this range the expected QE is larger than $85 \%$ and uniform over each pixel. For lower photon energies, in the 100-to- $250 \mathrm{eV}$ regime, the QE falls off and single photon counting with a high confidence in false positives discrimination will no longer be possible. However, PERCIVAL is expected to be usable under high fluxes down to $100 \mathrm{eV}$ and lower, in order to fully cover the FLASH operational energies. $^{2}$

At these relatively low energies, the attenuation lengths for both $\mathrm{Si}$ and $\mathrm{SiO}_{2}$ are on the order of $100 \mathrm{~nm}$, as shown in figure 1. The uppermost layer of a CMOS sensor, containing $\mathrm{SiO}_{2}$ and the metal lines of the circuitry, is in general several $\mu \mathrm{m}$ thick. Therefore, in order to achieve the aimed QE as well as a uniform response, passive entrance windows have to be reduced by orders of magnitude.

${ }^{2}$ After the last upgrade the photon energy can be tuned between 25 and $300 \mathrm{eV}$. 


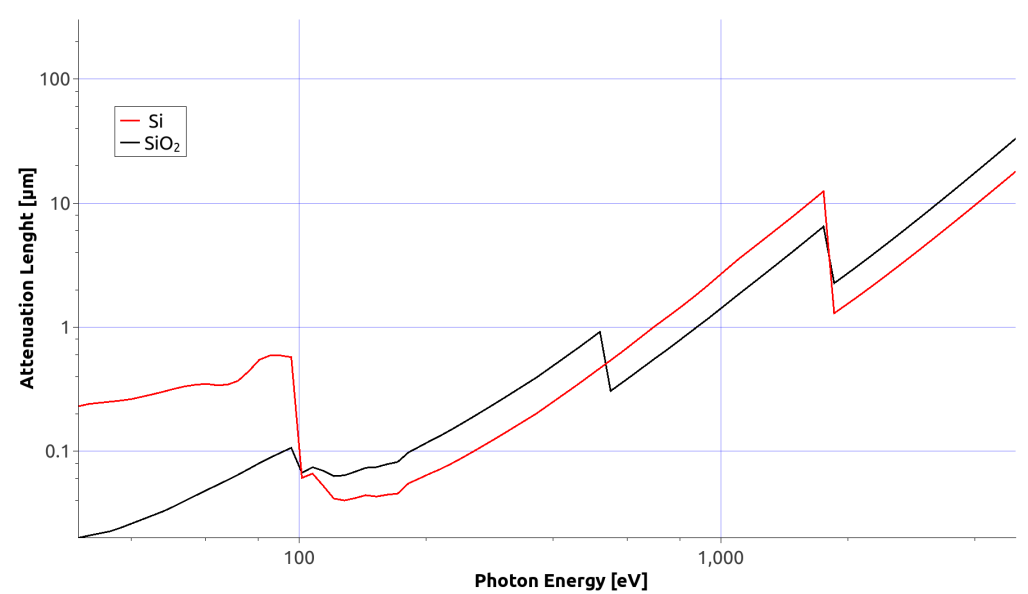

Figure 1. Attenuation lengths for $\mathrm{Si}$ and $\mathrm{SiO}_{2}$ versus photon energy [3]. For the low-end of the energy range to be covered by the PERCIVAL sensor, the attenuation length in both $\mathrm{Si}$ and $\mathrm{SiO}_{2}$ is significantly lower than $1 \mu \mathrm{m}$. Therefore a back-thinned process coupled with a back-illumination mode is required.

Back-illumination and back-thinning are thus essential to achieve the desired performance for soft X-rays.

Figure 2 illustrates two illumination modes: front-side-illuminated (FSI) where the photons arrive through the rather thick entrance window of circuitry, $\mathrm{SiO}_{2}$ and metals, (and therefore it could only provide reasonable QE for high photon energies); and back-side-illuminated (BSI). Here to achieve good performance for soft X-rays a regular FSI chip has to be back-thinned (in our case, from the original $700 \mu \mathrm{m}$ to $12 \mu \mathrm{m}$ ). This is done by NASA's Jet Propulsion Laboratory (JPL) applying their $\delta$-doping technique [4]. After this process, the entrance window is only a few nanometers thick.

In order to increase the spatial resolution, and therefore the level of detail of the scattering patterns to be recorded, high resolution in scatter angle (q) space is needed. This could be achieved with large pixels on a large area at a large distance. However, practical constraints (e.g. vacuum vessel size) push for moderate size sensors with (millions of) small pixels. Although the test sensors (to be described in section 2.2) have a pixel size of $25 \mu \mathrm{m}$, the final version of the chip will use 27 $\mu$ m pixels. ${ }^{3}$ Two different sensor sizes are foreseen: $2 \mathrm{M}$, with $1408 \times 1484$ pixels on $4 \times 4 \mathrm{~cm}^{2}$; and $13 \mathrm{M}$, with $3520 \times 3710$ pixels on $10 \times 10 \mathrm{~cm}^{2}$. To enable use in cloverleaf-like arrangements with 8 or 52 Megapixels, both will be 2-side buttable.

To cope with the rather large dynamic range needed (going from $10^{5}$ photons per pixel per frame to single-photon detection), PERCIVAL employs a lateral overflow approach to enable automatic gain adjustment on a per-pixel basis. The pixel schematic is shown in figure 3. It is based on the standard 3T structure (source-follower, reset, and select transistor) which is enhanced by the addition of a series of switches (SW0-2, AB) and capacitors ( $\mathrm{C} 0-\mathrm{C} 2)$. At low photon fluxes, during the charge integration, no current flows between the transistor and the capacitors and the system behaves as a regular 3T Active Pixel Sensor (APS). Switches SW0-2 and AB are biased at intermediate voltages. At higher fluxes, corresponding to a lower voltage on the diode, the current starts flowing through

${ }^{3}$ This modification was done to optimise the "Full Well" capacity. 

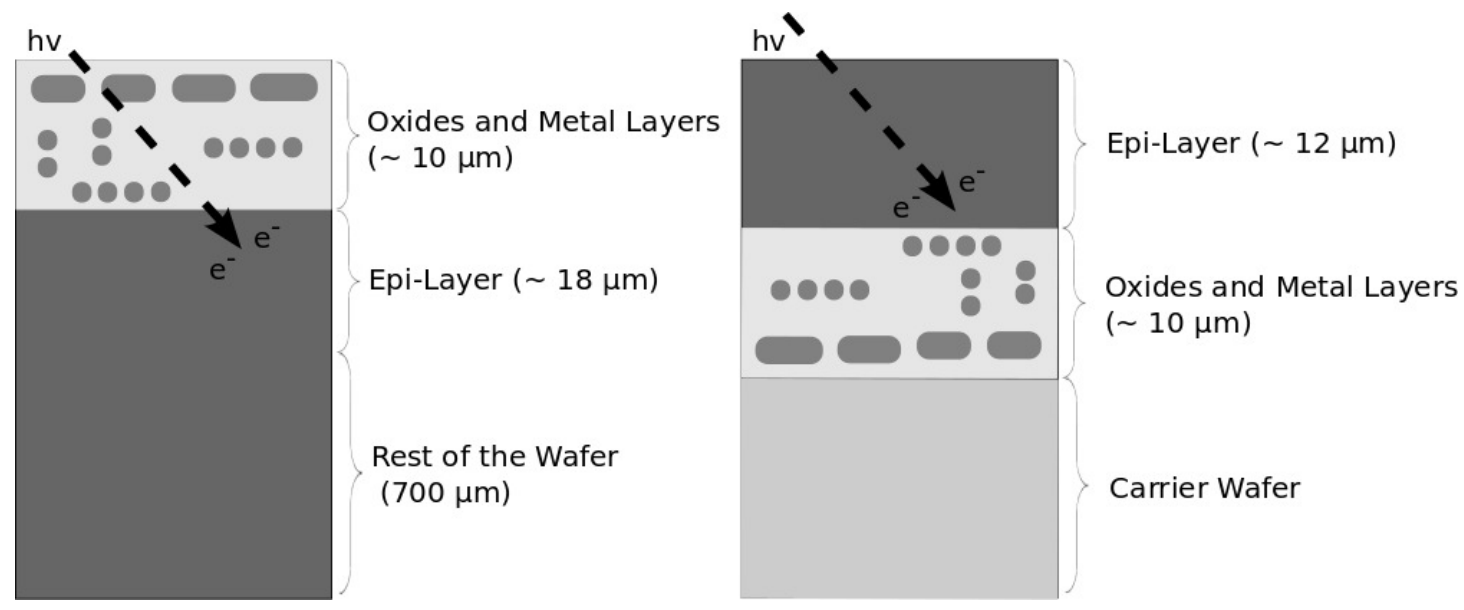

Figure 2. The two different operation modes of the Percival sensor. On the left, an FSI chip: impinging photons need to cross the entrance window consistent of metal layers and oxides. Most of the low-energy photons will be absorbed in this layer and lost. On the right, a BSI chip after back-thinning: the entrance window is only a few nanometers thick. Low-energy photons are now absorbed in a depleted region, increasing the efficiency.

SW0 charging the first additional capacitor. This process repeats when additional photons arrive charging the subsequent capacitors. During readout, the switches S0-2 are sequentially opened, the source-follower output compared to a threshold, and both ADC'd voltage and gain recorded for the best gain setting.

As a consequence, a "Full Well" capacity of $10^{7}$ electrons can be reached at the lowest possible gain, while in order to reach the goal of single-photon detection at low energies, in the highest gain the aimed noise level of around $15 \mathrm{e}^{-}$rms remains feasible, while the false positive probability is kept at $\sim$ value $10^{-6}$. Together, these two limits set an overall dynamic range of $10^{5}$ photons. The operational temperature has been, for the moment, kept at $-40^{\circ}$ degrees, awaiting detailed optimisation.

Each column is equipped with 7 ADCs (plus 1 spare). It consists of a coarse stage (that defines the 5 most significant bits) followed by a fine stage (that determines the 7 least significant bits). One bit is kept for the overrange and two more are used for the gain stage. In total, 15 bits per pixel need to be read out. To improve noise performance in the highest gain the sensor allows for digital Correlated Double Sampling (CDS) based on diode capacitance. To cope with the 3710 rows at the discussed frame rates $(120 \mathrm{~Hz}$ in the fastest mode), a conversion time of $7 \mu \mathrm{s}$ is needed. Data from 32 columns is multiplexed into one LVDS data output line running at $\sim 460 \mathrm{MHz}$ data rate. Altogether, 111 LVDS lines output the data from 24864 ADCs resulting in 50 Gbits/s image data from a single sensor running at $120 \mathrm{~Hz}$.

Two different operation modes can be used depending on the source: quasi-continuous illumination at a synchrotron or single-shot illumination at FELs. In both cases a modified rolling shutter scheme is applied. At a synchrotron, the fraction of a frame cycle in which the overflow capacitor is connected is maximised. Only during the short time used by the readout and reset, the pixel is not optimally sensitive to light. For each row-group this blind-time occurs at a different time. For FELs, the interesting photons arrive pulsed. Therefore the overflow is in operation only for a short 


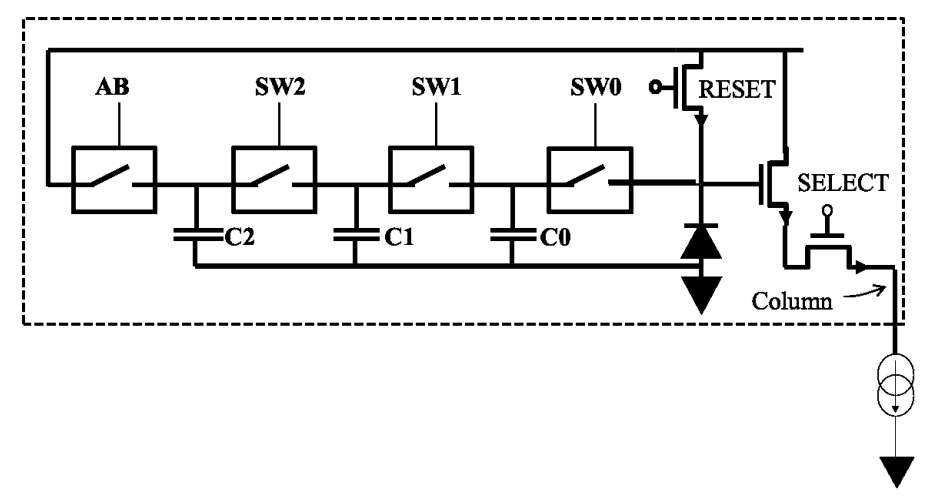

Figure 3. PERCIVAL pixel schematics. The components corresponding to a regular "3T pixel" are coupled with a series of capacitors and switches to increase the dynamic range. Additionally, anti-blooming overflow components are also shown.

period of time around the arriving of the FEL pulse. As has already been mentioned, the base repetition rate at today's FELs ranges from 10 to $120 \mathrm{~Hz}$.

\subsection{Prototype sensors}

In order to evaluate the performance of various pixel architectures, the gain decision logic, and the ADCs, small test sensors were produced. Sensor size is $210 \times 160$ pixels of $25 \times 25 \mu \mathrm{m}^{2}$ and it allows comparison of 6 different pixel types while the rest of the on-chip components were kept identical. Two different version were fabricated: TS1.0 (which implemented 6 variations of an annular photo-diode design [5]) and TS2 (where pixels are based on a more conventional n-well design). In each of these two chips, three of the six pixel types have the capacitors needed for gain switching (right-hand side), while the other three implement only the 3T design (left-hand side).

Early tests of the TS1.0 and TS2 revealed a higher-than-expected leakage current. To understand and mitigate this effect, an additional test device was conceived varying the pixel design: TS1.1. As it is explained in section 3, whereas the leakage current was decreased in thick-oxide variants of the pixels, the noise values obtained with this device were still above the performance aims. An investigation of the noise contributions in TS1.0 unveiled that a large part of the excess of noise originated at the ADC. Therefore, improved ADC in combination with and additional amplifier stage were designed and implemented in a new test prototype: TS1.2. This device is currently under study.

\section{Testing PERCIVAL prototypes}

In [6], the results of the first TS1 BSI chip were presented. Noise maps and images of Airy patterns were shown. It was found that the light response variation between different pixel types was larger than expected. In particular, low-energy photon sensitivity was very low for some pixel types which points to charge being collected only from a part of the sensor volume. After preliminary analysis, a potential source of contamination was discovered in the chamber where the back-thinning process took place. Once the problem was solved, another wafer underwent BSI processing. A comparison between the old BSI and the new BSI is made in the next sections. 


\subsection{Noise}

To evaluate the noise performance of the PERCIVAL chips, the Photon Transfer Curve [7] (PTC) technique was used. ${ }^{4}$ The system gain and therefore the conversion from measured ADU to $\mathrm{e}^{-}$rms can be derived from the relation of noise and signal at various illumination levels.

The noise levels for the TS1.0 pixel types lie in the range of 23 to $25 \mathrm{e}^{-}$for the 3T pixels when working in synchrotron mode and between 20 and $24 \mathrm{e}^{-}$when using the FEL mode. In the case of pixels coupled with the gain switching circuitry the values range from 25 to $28 \mathrm{e}^{-}$and from 23 to $27 \mathrm{e}^{-}$for the two modes.

No significant difference was found between the old and the new BSI when analysing the noise levels. To decrease these levels, an upgraded chip, TS1.2, with improved ADCs (believed to generate the largest contribution to the noise) and an additional amplifier stage has been designed. Recently received from the foundry, noise tests are ongoing.

\subsection{Uniform illumination}

The two BSI TS1.0 chips were compared by measuring the average of a series of images under uniform illumination applying CDS as well as dark frame subtraction. A LED providing uniform illumination with red optical photons was used as a source. Sensor temperature was set at $-40{ }^{\circ} \mathrm{C}$. Results are shown in figure 4.

In the case of the old chip, signals were observed to be very similar for 4 out of the 6 different pixel types while 2 showed significantly higher response to the illumination. Results for the new chip, show a different behaviour. A much more uniform signal is obtained over the whole sensor surface. However, pixels at on side of a the boundary between the different pixel regions show lower signals whereas neighbouring pixels show higher signals. This effect, as if these pixels were "stealing" charge from their neighbours, is visible at most pixel type boundaries. It hints at differences in the pixels' capability to collect charge, quite possibly in particular from the regions of the epilayer more distant from the circuitry. Such an effect is also visible at the edge of the sensor.

\subsection{Sensitivity}

To establish the sensitivity of the PERCIVAL sensor to low-energy photons, campaigns at different light sources have taken place. To obtain a quick and clear confirmation of the dominant photon energy observed, the sensor is exposed to photons through a pinhole situated at a certain distance from the sensor. Circular symmetric patterns (known as Airy disks) are the result of diffraction at the pinhole and can be measured. Ring radii are characteristic for the photon energy at a given setup geometry, enabling photon energy confirmation.

Images of these Airy patterns were measured with the old chip in the TwinMic beamline at Elettra Light source, as explained in [2] and [8]. A clear difference between the response from the different pixel types was visible. The broader 1st-harmonic disks were visible only in two pixel types. In all other pixel types, contributions from higher harmonic dominated. Sensitivity of pixel types A, B, C and F to low-energy photons was clearly compromised.

\footnotetext{
${ }^{4}$ The PTC is constructed by plotting the $\log 10$ average signal vs. the $\log 10$ of the sigma for illumination with optical photons at a wide range of intensities. On the assumption that the region that falls under a slope of 0.5 implies Poisson-dominated statistics.
} 

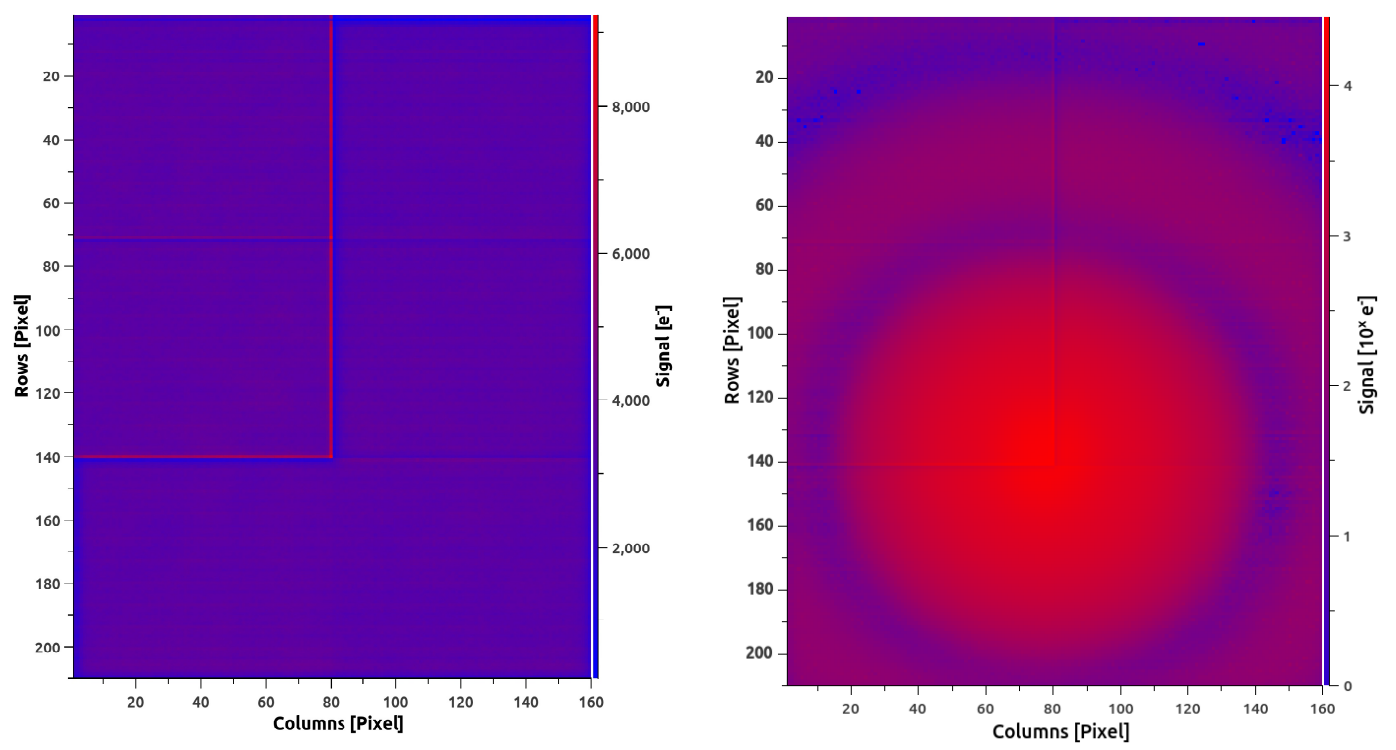

Figure 4. Average Correlated Double Sampling (CDS) of a series of images. On the left, under uniform (visible) illumination, the new chip shows a uniform response over the whole area. As previously said, on the left half of the frame pixels are provided with the gain switching capacitors whereas on the right half only standard 3T structure is used. In addition, a higher signal was observed at the boundaries between the different pixel types. On the right, images of Airy disk patterns generated by a nominally $5 \mu$ m pinhole placed upstream of the sensor recorded with the new chip at Diamond's I-10 beamline. Nominal energy was $400 \mathrm{eV}$. Careful analysis of the recorded image pattern revealed no sign of higher harmonic contents in the data.

The same experiment was conducted with the new chip at Diamond's I-10 beamline. At this beamline a signficantly lower contribution of higher harmonics than at TwinMic is expected. A $5 \mu \mathrm{m}$ pinhole was placed $2.4 \mathrm{~m}$ from the sensor. The nominal energy was $400 \mathrm{eV}$. The resulting image can be seen in figure 4 , on the right-hand side. Signal differences between the different pixel regions are much lower.

Overall, a much more uniform signal can be obtained with the new chip compared to the old one. Also, and after a careful analysis of different Airy disks throughout the whole chip and a comparison with the expected patterns for the specific parameters used (pinhole diameter, distance pinhole-sensor, photon energy), no evidence of higher harmonics was found in the image data.

\subsection{Charge Collection Efficiency}

The absence of higher harmonics at I-10 (nominally $400 \mathrm{eV}$ ), enables the I-10 data to be used to obtain a first estimation of the PERCIVAL sensor Charge Collection Efficiency (CCE) at this energy. This figure of merit is defined as follows:

$$
\mathrm{CCE}=\frac{N_{e^{-}}}{N_{\mathrm{ph}}}
$$

where $N_{e^{-}}$is the number of carriers measured with the PERCIVAL sensor and $N_{\mathrm{ph}}$ is the number of carriers produced by an impinging photon flux, $\phi_{\mathrm{ph}}$. In Silicon, an average of $3.6 \mathrm{eV}$ are needed 


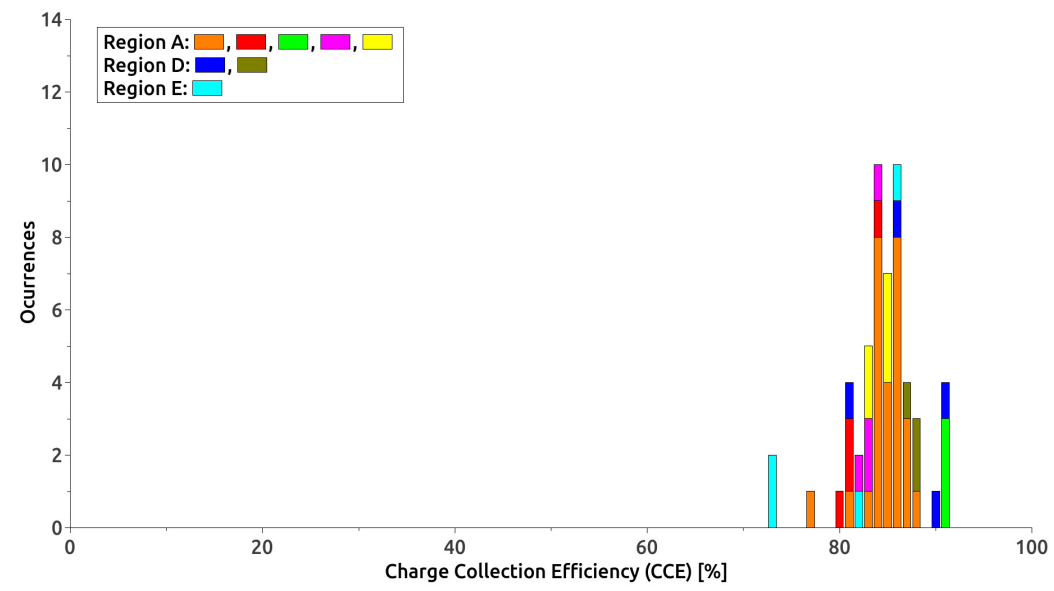

Figure 5. Several measurements of the CCE of the new sensor are summarised in this histogram. Each entry was obtained by averaging the CCE of more than 1000 pixels of a given pixel type obtained during a single measurement. The CCE for 3 different pixel types (A,D, and E) were measured using between 3 and over 20 independent CCE datasets per pixel region. The impinging photon-energy was $400 \mathrm{eV}$ and the photon flux, $\phi_{\mathrm{ph}}$, estimated with a well-calibrated photo-diode. The mean value was found around $85 \%$.

to create a electron-hole pair in the most probable interaction. Thus, for $400 \mathrm{eV}$ photons, 111,11 carriers per photons will be generated.

A well-defined $\phi_{\text {ph }}$ is crucial to CCE measurements. At I-10, this was determined by a PTBcalibrated photo-diode. It was placed $\sim 1 \mathrm{~m}$ in front of the PERCIVAL sensor. The size of the beam was limited with a set of slits so that all photons arriving at the sensor could be also measured with the photo-diode. Measurements of the photo-diode and the PERCIVAL sensor were interleaved.

A histogram was obtained by averaging the CCE of more than 1000 pixels of a given pixel type obtained during a single measurement. The CCE for 3 different pixel types were measured using between 3 and over 20 independent CCE datasets per pixel region. It is shown in figure 5. Most of the measurements were done in pixel region $\mathrm{A}$, but points coming from regions $\mathrm{D}$ and $\mathrm{E}$ are also included. The resulting distribution shows a mean value around $85 \%$.

This measurement of the CCE constitutes a lower limit for the QE. To have an estimation of this latter parameter, a measurement with detection of single photons is required.

\section{Summary and outlook}

A collaboration between different Photon Science institutions is developing the CMOS-based PERCIVAL soft X-ray imager. A large Quantum Efficiency (QE) over a wide range of photon energies, small pixel size allowing good spatial resolution, FELs base-rate compatible, (multi-)megapixel arrangements 2-side buttable and large dynamic range at a low noise are key performance aims.

Small test sensors $(160 \times 210$ pixels $)$ have been designed and tested. Previously a backthinned, BSI chip was tested and it showed large variations between the different pixel regions and, for some of these regions, low sensitivity to low-energy photons. It is thought that this was due to a contamination during the back-thinning process. New chips were produced and tested. 
A more much uniform response was obtained under uniform optical illumination for the new chip, and sensitivity to $400 \mathrm{eV}$ photons has been proven for all pixel types. Moreover, and after a full evaluation of the high harmonic contribution and the measurement of the impinging photon flux, an estimation of the PERCIVAL sensor Charge Collection Efficiency (CCE) was done. The resulting value of $85 \%$ represents a lower limit to the $\mathrm{QE}$ at $400 \mathrm{eV}$. Furthermore, it is compatible with the aimed parameters previously discussed for this photon energy.

A more detailed analysis of the CCE is now foreseen by scanning the energy range. In addition, an estimation of the PERCIVAL sensor QE is envisioned. To do so, a single-photon measurement is required and thus, a lower noise level imperative. In that context, a test campaign of the TS1.2 chip have just started.

\section{References}

[1] J. Becker et al., Challenges for the Adaptive Gain Integrating Pixel Detector (AGIPD) design due to the high intensity photon radiation environment at the Europeal XFEL, PoS(VERTEX 2012)012.

[2] C.B. Wunderer et al., The PERCIVAL soft X-ray imager, 2014 JINST 9 C03056

[3] Center for X-ray Optics (CXRO), X-ray data base (2015).

[4] B.C. Jacquot et al., Direct detection of 100-5000 eV electrons with delta-doped silicon CMOS and electron-multiplying CCD imagers, IEEE T. Electron Dev. 59 (2012) 1988.

[5] A. Lahav, R. Reshef and A. Fenigstein, Enhanced X-ray CMOS sensor panel for radio and fluoro application using a low noise charge amplifier pixel with a partially pinned PD, talk given at the 2011 International Image Sensor Workshop, June 8-11, Hakodate, Japan, (2011).

[6] C.B. Wunderer et al., The PERCIVAL soft X-ray imager, 2015 JINST 10 C02008.

[7] J.R. Janesick, photon transfer $D N \rightarrow \lambda$, SPIE Press (2007).

[8] C.B. Wunderer et al., PERCIVAL: an international collaboration to develop a MAPS-based soft X-ray imager, Synchrotron Radiat. News 27 (2014) 30. 